Supporting Information for

\title{
Improving the Solubility and Bioavailability of Apixaban via Apixaban-Oxalic Acid Cocrystal
}

Yong Chen, Long Li, Jia Yao, Yu-Yu Ma, Jia-Mei Chen* and Tong-Bu Lu*

Table S1. Mean pharmacokinetic parameters of free apx and apx-oxa cocrystal in male beagle dogs.

\begin{tabular}{|c|c|c|c|c|c|c|c|}
\hline Group & Dosage & Sex & Compound & Subject & $\begin{array}{c}\text { Tmax } \\
\text { (h) }\end{array}$ & $\begin{array}{c}\text { Cmax } \\
(\mathrm{ng} / \mathrm{ml})\end{array}$ & $\begin{array}{l}\text { AUClast } \\
\left(\mathrm{h}^{*} \mathrm{ng} / \mathrm{ml}\right)\end{array}$ \\
\hline \multirow[t]{6}{*}{ IG1 } & 1 & Male & Apx (form N-1) & 1 & 2 & 410 & 3300 \\
\hline & & & & 2 & 2 & 570 & 4100 \\
\hline & & & & 3 & 2 & 340 & 3100 \\
\hline & & & & Mean & 2 & 439 & 3500 \\
\hline & & & & SD & 0 & 120 & 530 \\
\hline & & & & $\mathrm{CV} \%$ & 0 & 26 & 15 \\
\hline \multirow[t]{6}{*}{ IG2 } & 1 & Male & Apx-oxa & 4 & 4 & 860 & 8000 \\
\hline & & & & 5 & 2 & 1800 & 13000 \\
\hline & & & & 6 & 1 & 960 & 7700 \\
\hline & & & & Mean & 2.33 & 1220 & 9720 \\
\hline & & & & SD & 1.5 & 540 & 3200 \\
\hline & & & & $\mathrm{CV} \%$ & 65 & 44 & 33 \\
\hline
\end{tabular}




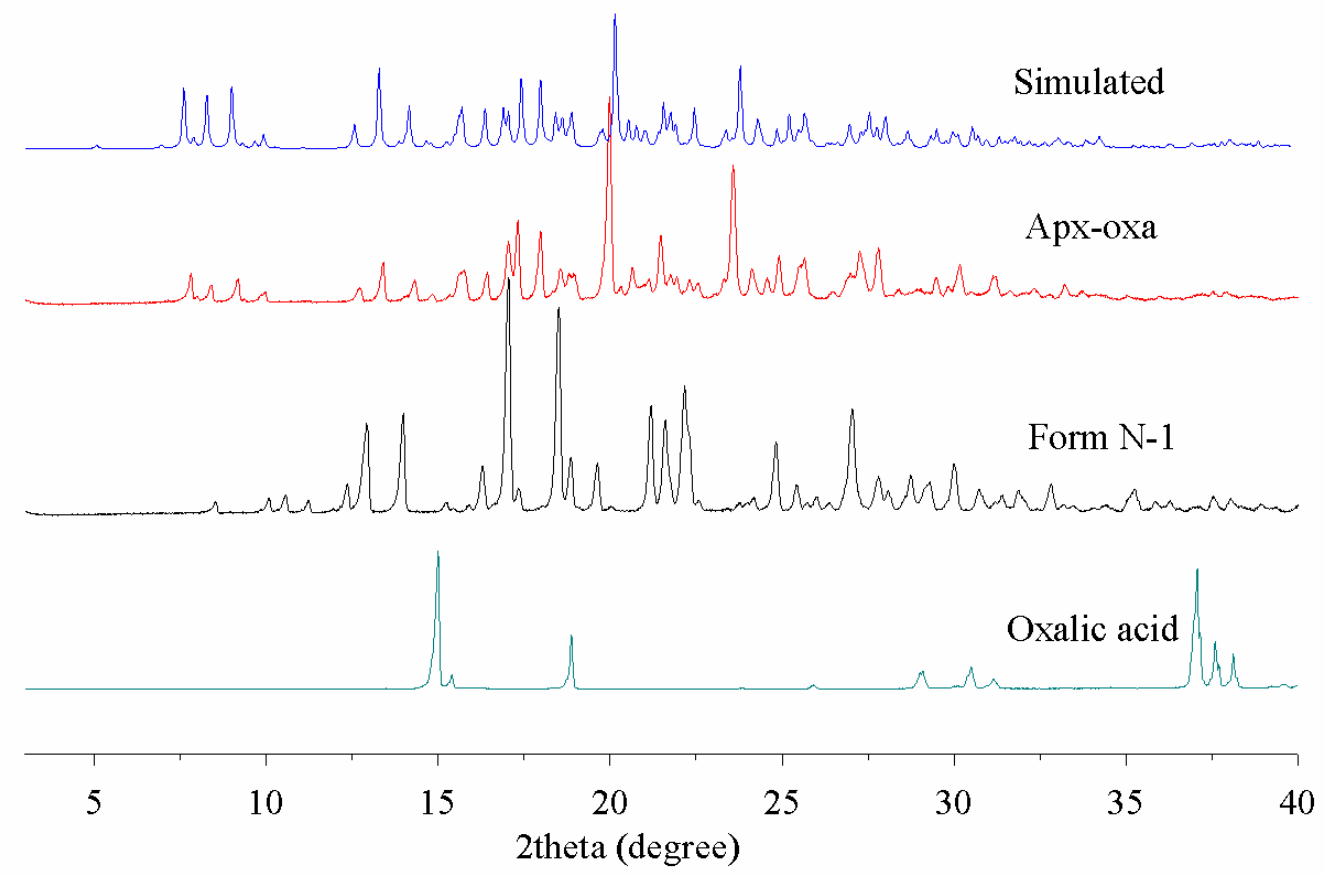

Figure S1. PXRD patterns for oxalic acid, apx form N-1 and apx-oxa cocrystal.

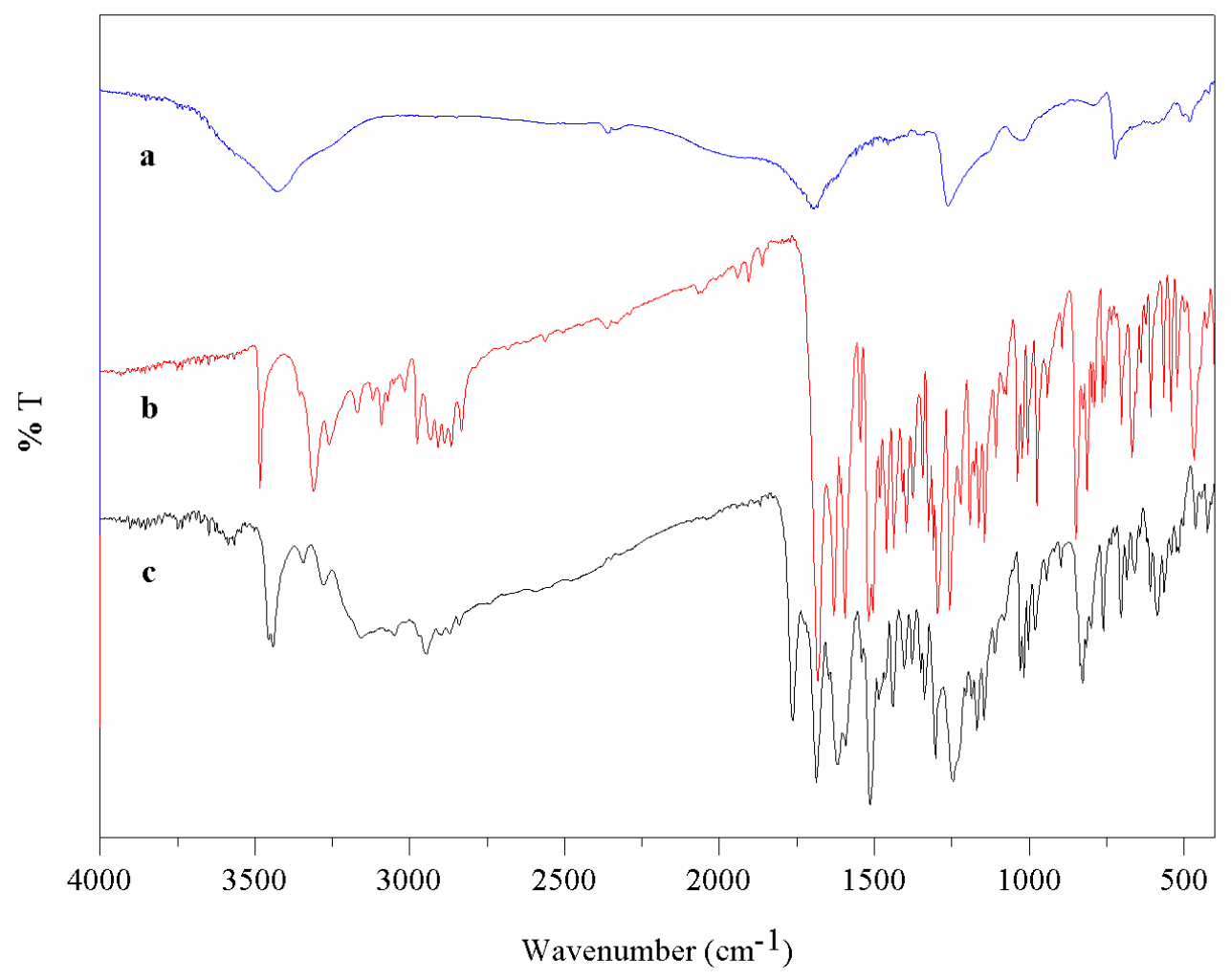

Figure S2. FTIR spectra for (a) oxalic acid, (b) form N-1 and (c) apx-oxa cocrystal. 


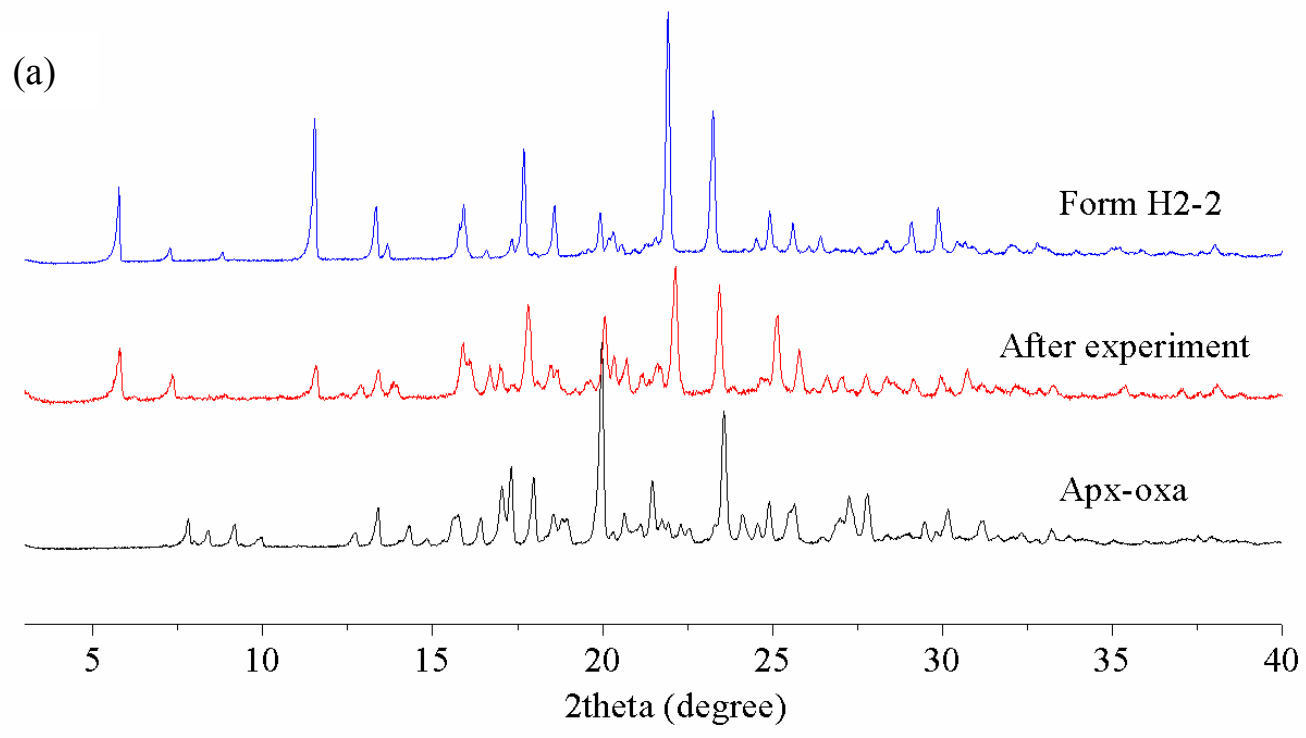

(b)

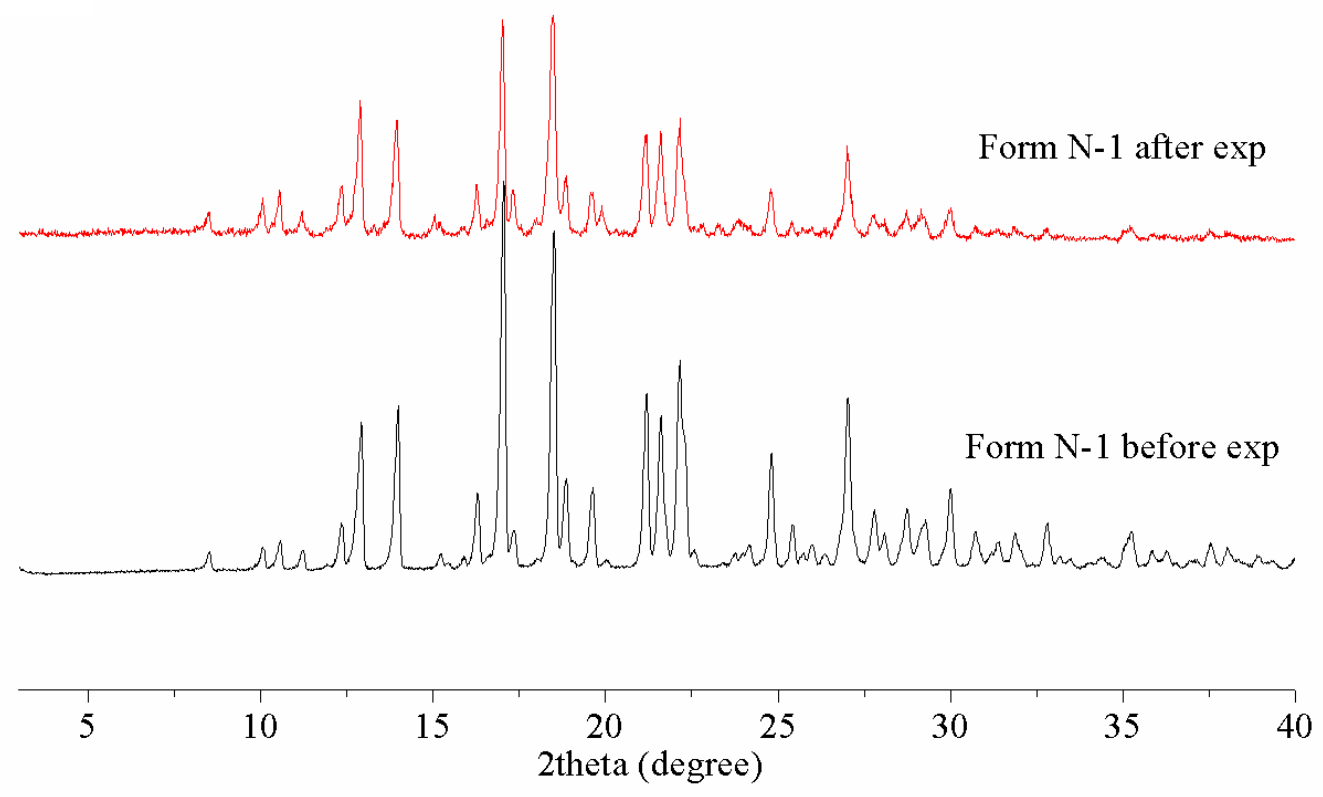

Figure S3. PXRD patterns for (a) apx-oxa cocrystal, and (b) form N-1 before and after the powder dissolution experiments. 


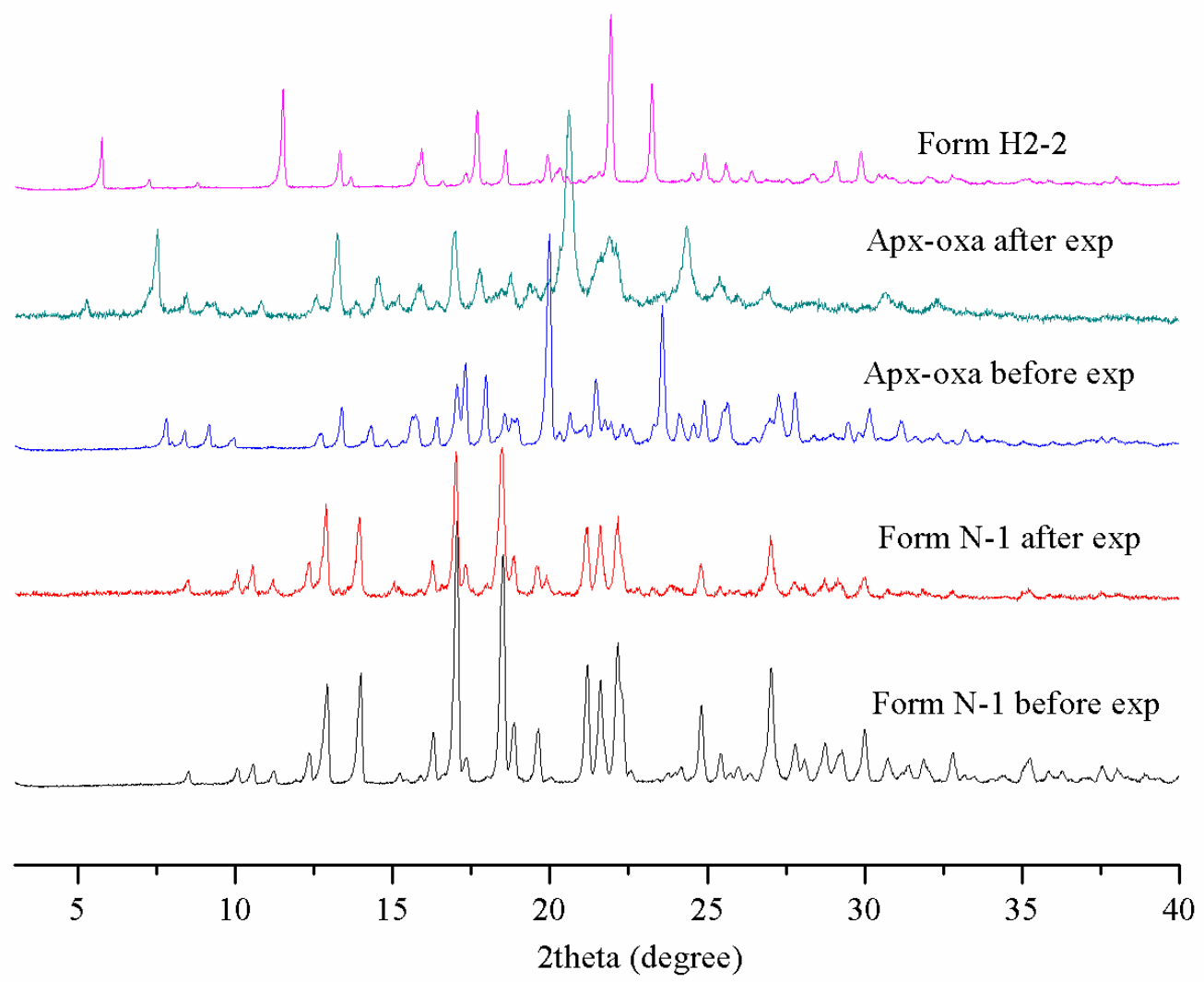

Figure S4. PXRD patterns for form N-1 and apx-oxa cocrystal before and after IDR experiments. 